\title{
Estimation of the radioactive source dispersion from Fukushima nuclear power plant accident
}

\author{
Michael Schöppner ${ }^{\mathrm{a}, \mathrm{b}}$, Wolfango Plastino ${ }^{\mathrm{a}, \mathrm{b}, *}$, Pavel Povinec ${ }^{\mathrm{c}}$, Mika Nikkinen ${ }^{\mathrm{d}}$, \\ Federico Ruggieri ${ }^{\mathrm{b}}$, Francesco Bella ${ }^{\mathrm{a}}$ \\ a University of Roma Tre, Department of Mathematics and Physics, Via della Vasca Navale 84, I-00146 Roma, Italy \\ ${ }^{\mathrm{b}}$ INFN, Section of Roma Tre, Via della Vasca Navale 84, I-00146 Roma, Italy

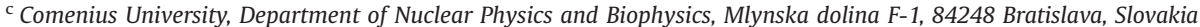 \\ ${ }^{\mathrm{d}}$ Preparatory Commission for the Comprehensive Nuclear Test-Ban-Treaty Organization (CTBTO), Provisional Technical Secretariat, Vienna International \\ Centre, PO Box 1200, 1400 Vienna, Austria
}

\section{H I G H L I G H T S}

- Atmospheric transport modelling is used to characterize the Fukushima source term.

- The emissions are reconstructed according to 133-Xe detections in Japan and China.

- Correlation of the estimated source terms to 137-Cs and 131-I detections.

\section{A R T I C L E I N F O}

Available online 2 April 2013

\section{Keywords:}

Fukushima

Atmospheric transport modeling

Xenon

Source term

\begin{abstract}
A B S T R A C T
Following the Fukushima nuclear power plant accident detections of ${ }^{133} \mathrm{Xe}$ have been made in various locations. Using results of these remote measurements the Fukushima ${ }^{133} \mathrm{Xe}$ source term has been reconstructed and compared with previously reconstructed ${ }^{137} \mathrm{Cs}$ and ${ }^{131}$ I source terms. The reconstruction is accomplished by applying atmospheric transport modeling and an adapted least square error method. The obtained results are in agreement with previous estimations of the Fukushima radionuclide source, and also serve as a proof of principle for source term reconstruction based on atmospheric transport modeling.
\end{abstract}

(c) 2013 Elsevier Ltd. All rights reserved.

\section{Introduction}

The Tohuku earthquake on 11 March 2011 and the following tsunami at the north-eastern coast of Japan resulted in the worldwide known nuclear accident at the Fukushima Nuclear Power Plant (NPP). The state of several reactors worsened during the following days and large amounts of radioactive material were released into the environment, i.e. land, sea and atmosphere. The assessment of the regional and global impact depends on the knowledge of which radionuclides had been emitted in what quantities. Also the time development is of importance as it determines with which prevailing winds or sea currents the radioactive material is dispersed. Due to the severe conditions on-site it has not been possible to determine the source term directly. Information about the time-line of events can be found on the website of the International Atomic Energy Agency (IAEA, 2011).

\footnotetext{
* Corresponding author at: University of Roma Tre, Department of Mathematics and Physics, Via della Vasca Navale 84, I-00146 Roma, Italy. Tel.: +3906 5733 7277; fax: +3906 57337102 .

E-mail address: plastino@fis.uniroma3.it (W. Plastino).
}

The International Monitoring System (IMS), which is currently built up by the Comprehensive Nuclear-Test-Ban Treaty Organization (CTBTO), continuously takes environmental measurements including atmospheric concentrations of certain radionuclides. The IMS design incorporates 80 radionuclide monitoring stations worldwide, of which 40 stations can also detect radioactive noble gases such as radioxenon isotopes (Zähringer et al., 2009). Radioxenon is mainly emitted by NPPs and other nuclear facilities, such as medical isotope production facilities, which create a global radioxenon background with regional dependence (Wotawa et al., 2010). In the days and weeks following the Fukushima accident the concentrations of several radionuclides including ${ }^{133}$ Xe raised at multiple IMS stations throughout the world (Stohl et al., 2011).

\section{Methods}

The time-dependent relationship between a source that is emitting particles and a receptor in a remote location can be described with a source-receptor sensitivity (SRS) matrix. To determine the sensitivity between the Fukushima NPP and the affected IMS stations, Atmospheric Transport Modelling (ATM) 


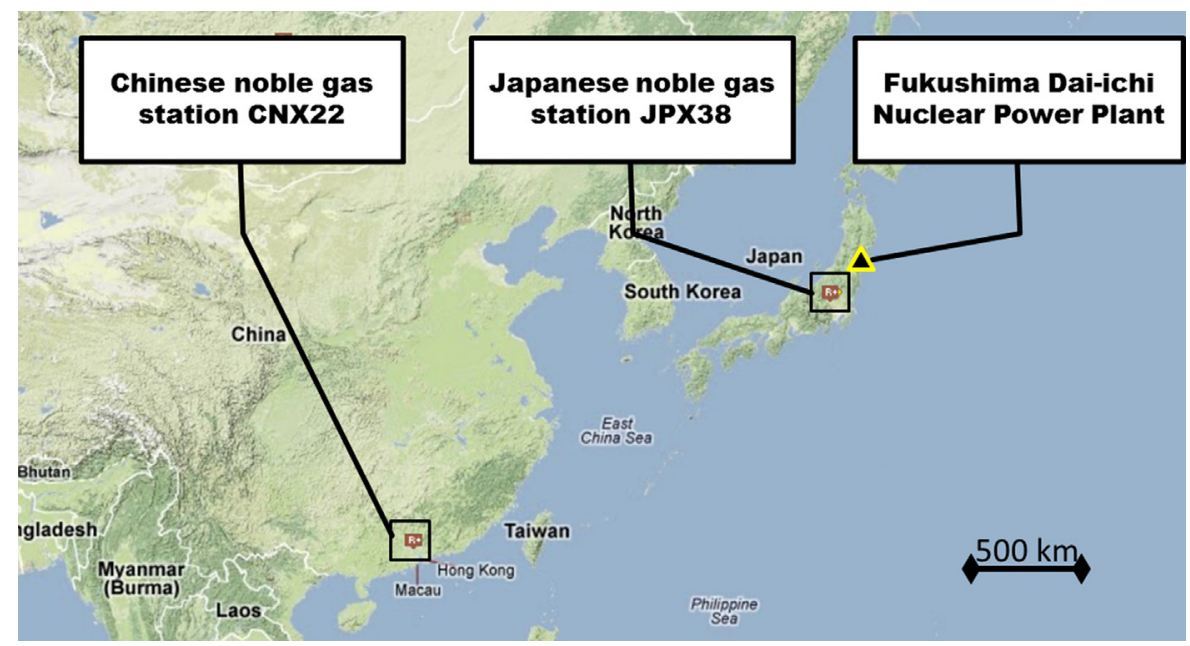

Fig. 1. Map of East Asia with Fukushima and the two noble gas monitoring stations JPX38 and CNX22. (www.ctbto.org).

has been utilized. The general purpose of ATM is to calculate the sensitivity, which determines the relation between two points on a spatial grid. The concentration $c$ at the receptor is calculated from the source emission strength $S$ at coordinates $i, j$ during the time interval $n$, and the accordant source-receptor sensitivity $M$ :

$c=M_{i j n} S_{i j n}$

when sources from more than one location or more than one time interval are contributing to the concentration, summing over all sources and time intervals gives the total concentration (Wotawa et al., 2003). The Lagrangian Particle Dispersion Model FLEXPART uses the zero-acceleration scheme

$X(t+\Delta t)=X(t)+v(X, t) \Delta t$

to integrate the trajectory equation. Various radionuclide-loss processes such as radioactive decay and dry and wet deposition have been included in the simulations. However, radioxenon and noble gases in general, which will be mainly discussed in this paper, are not affected by deposition effects (Stohl et al., 2010). Due to its many parameters ATM is always subject to uncertainties that are not trivial to quantify directly. However, the applied FLEXPART model has been validated against several meso-scale tracer experiments in order to ensure its capability (Stohl et al., 1998) and was found to produce good correlation with no particular bias. Effects of local meteorological patterns that are not resolved by ATM can be analysed with a statistical time series analysis (Plastino et al., 2010).

The Flexpart model has been ported to the local grid computer at the University of Roma Tre/INFN. It is running with atmospheric data from the European Centre for Medium-Weather Forecast (ECMWF) in a spatial resolution of one degree latitude/longitude and time resolution of $3 \mathrm{~h}$.

The influence of the worldwide ${ }^{133} \mathrm{Xe}$ background on a certain detector can be simulated with a radioxenon emission inventory that describes average emissions from legitimate radioxenon sources (Kalinowski and Tuma, 2009). Emissions from a certain grid point and time can reach the same detector multiple times due to various particle trajectories following the wind dispersion. The concentration during one detection period of the receptor can also be composed from emissions from multiple emissions in time and space. However, when assuming one single emitter with a known location (as it is shown later), information about the source term can be deducted from the remote measurements.

The virtual Data Exploitation Centre (vDEC), established by the CTBTO, supplies IMS data for research purposes including radioxenon concentrations detected following the Fukushima accident. When choosing detection data to reconstruct the source term, spatial vicinity and temporal availability of data are important. Here, data from the Japanese station JPX38 in Takasaki close to Tokyo and about $250 \mathrm{~km}$ from Fukushima, and from the Chinese station CNX22 near Hong Kong about $3000 \mathrm{~km}$ from Fukushima have been considered, see Fig. 1. For the Japanese station elevated radioxenon data are only available for the time period from 05 April 2011; for the Chinese station radioxenon data are available after 25 March 2011.

When a constant source term is assumed it results in a particular time series of concentrations at the receptor. When compared to the experimental detection data the simulated time series most likely possesses different features and the sum of errors can be calculated. Due to the usual distribution over more than one order of magnitude it is useful to calculate the sum of square errors on a logarithmic scale. When changing the time dependence of the source term, the simulated time series at the detector changes according to the source-receptor sensitivity matrix, and with these changes also the sum of square errors changes. The source term can be adjusted in a way to minimize the summed log-scale square errors between simulated and experimental data, maximizing the alikeness between both of them.

The experimental ${ }^{133} \mathrm{Xe}$ data has been taken in collection intervals of twelve hours with Swedish Automatic Unit for Noble gas Acquisition (SAUNA) systems. Therefore, when reconstructing the Fukushima source term, a time resolution of $12 \mathrm{~h}$ has also been used. SAUNA systems detect noble gases with beta-gamma coincidence measurements (Auer et al., 2010). The Minimum Detectable Concentration is in the range $0.2-0.4 \mathrm{mBq} / \mathrm{m}^{3}$. The average error for the used data set of radioxenon detections is $10 \%$.

\section{Results and discussion}

Prior to the Fukushima nuclear accident, the Japanese CTBTO station detected ${ }^{133} \mathrm{Xe}$ emitted from multiple NPPs in the order of $0.1-1 \mathrm{mBq} / \mathrm{m}^{3}$. However, during the days following the accident no data were available. Data taking restarted on 4 April 2012, showing ${ }^{133} \mathrm{Xe}$ concentrations of the order of $0.1-1 \mathrm{~Bq} / \mathrm{m}^{3}$ (Fig. 2). The legitimate background that is produced by operating NPPs and medical isotope production facilities can be reproduced by ATM and the standard radioxenon emission inventory (Kalinowski and Tuma, 2009). When compared to the pre-Fukushima detections, as presented in Fig. 2, the simulations depict the background data.

For the Chinese station the data are only available from 25 March, where a similar pattern is observed, when the level of 


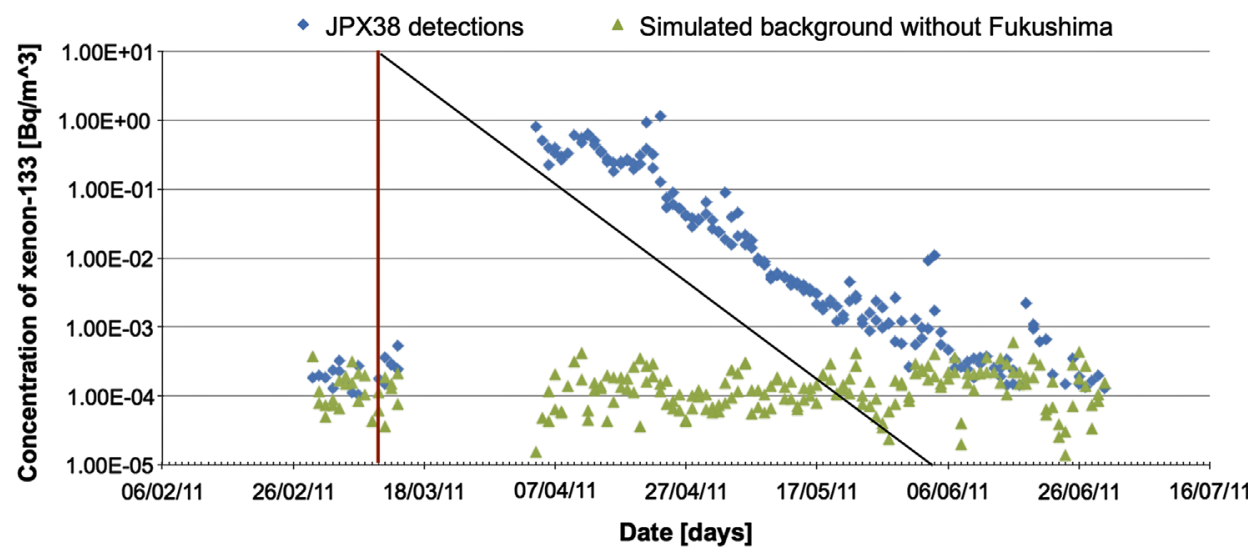

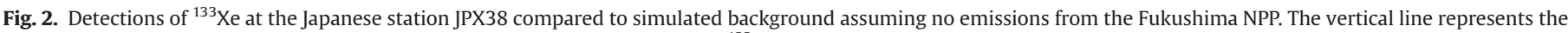
first day of the Fukushima accident; the sloped line represents the half-life of ${ }^{133}$ Xe. No data were taken between 15 March and 3 April.

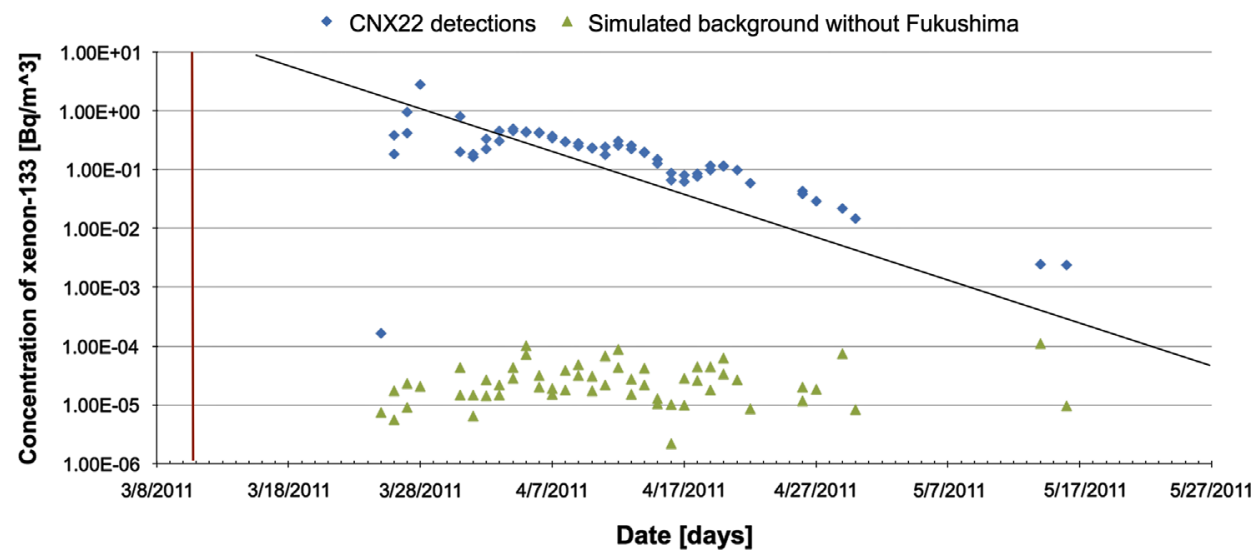

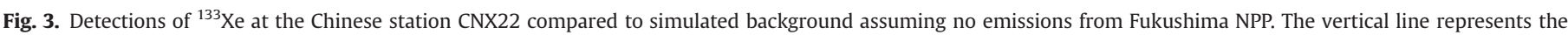
first day of the Fukushima accident; the sloped line represents the half-life of ${ }^{133}$ Xe. The data were only available from 25 March 2011.

concentration has increased on 26 March 2011 from the background levels of about $0.1 \mathrm{mBq} / \mathrm{m}^{3}$ to elevated levels of $0.1-1 \mathrm{~Bq} / \mathrm{m}^{3}$, (Fig. 3).

In both cases the ${ }^{133} \mathrm{Xe}$ levels raised by two to three orders of magnitude following the Fukushima nuclear accident. From this change it is concluded that Fukushima NPP can be handled as the only significant source of ${ }^{133} \mathrm{Xe}$. It is also possible that the detectors of the two stations have been contaminated due to high radioactive concentrations and that a memory effect could be responsible for part of the time series. In both stations a particular decline in the ${ }^{133} \mathrm{Xe}$ concentration has been similar to its decay rate. The analysis of this particular decline is out of scope of this work, but it has to be remembered that a memory effect would lead to an overestimation of the source term.

Due to the time offset between the Fukushima accident and the available detection data, as well as the possibility of detectors contamination, only the first days of data collection have been used to reconstruct the Fukushima source term. Especially the downtime and the late re-start of the radioxenon detector at the Japanese station allow only the source term reconstruction from Japanese data for 22 March and later. Anyway, from the Chinese noble gas detector it was possible to deduct information about the source term from 13 March and later. Gaps in the reconstructed source term are due to unfavourable meteorological conditions, i.e. when according to the simulation the prevailing winds did not allow to establish a sensitivity between source and receptor.

The time-dependent source terms of ${ }^{131} \mathrm{I}$ and ${ }^{137} \mathrm{Cs}$ following the Fukushima accident have been established previously with a similar method (Schoeppner et al., 2011). Thus, it is possible to compare how the Fukushima source term is seen by different stations and isotopes. From the previous results the ${ }^{137} \mathrm{Cs}$ and ${ }^{131} \mathrm{I}$ source terms have been overestimated, and therefore downscaled to fit more recent estimations of $3.5-27 \mathrm{PBq}$ for ${ }^{137} \mathrm{Cs}$ and 152-160 PBq for ${ }^{131}$ I (Hirose et al., 2012).

Fig. 4 shows the source term as seen according to four different variants of radionuclides and stations, and the applied method of minimization. Two distinguished periods can be identified in the reconstructed time series. One is from 12 March to 22 March. The second period is from 28 March to 9 April. The first period shows a correlation of the ${ }^{133} \mathrm{Xe}$ source term as seen from the Chinese station with the ${ }^{137} \mathrm{Cs}$, and the ${ }^{131}$ I source term as seen from the Japanese station.

For the second period only ${ }^{133} \mathrm{Xe}$ data is available. The both variants of the source terms are showing the same orders of magnitude values, and they share a characteristic decline from 1 April to 3 April 2011. After the decline, the concentrations of both variants show an antilinear dependence, and go apart for 4 and 5 April.

The differences in the correlation between the two periods can have various reasons. First, during the first period the Fukushima source was certainly more active than during the second period, and therefore the first period should bear sharper characteristics that are easier to identify. The previously mentioned possible contamination of the detectors may also be responsible for a decreased precision of later detections from which the second period has been reconstructed.

The deducted source terms can be compared with the time-line of events at the site of the Fukushima NPP. Since the on-site situation at the Fukushima NPP has prevented a direct assessment of radioactive emissions, only external observations have been 


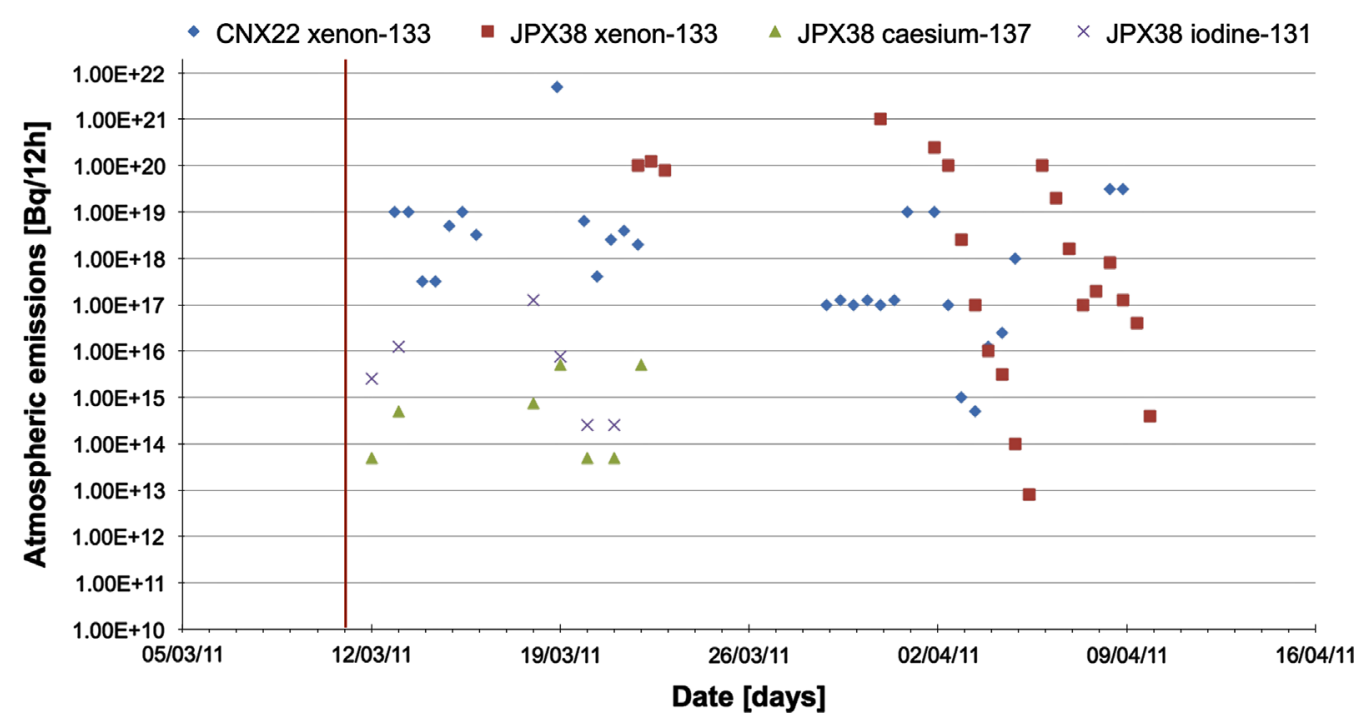

Fig. 4. The Fukushima source term as reconstructed from the CTBTO radionuclide stations and registered radionuclides.

taken into account. Multiple explosions were reported on 12, 14 and 15 March (IAEA, 2011). Large emissions of smoke were observed on 18 March, with lower amounts on the following days. The results from the combined simulations fit into this picture. Peaks on 13 and 15 March coincide with the reported explosions. Also the peak on 18 and 19 March goes along with the smoke observations and the decrease of smoke in the following days. The magnitude of the two presented ${ }^{133} \mathrm{Xe}$ source terms (Fig. 4) is comparable with estimated releases of 13.4-20.0 EBq in Stohl et al. (2011).

\section{Conclusions}

The Fukushima ${ }^{133} \mathrm{Xe}$ emissions have affected detectors in medium distances of several hundred to three thousand kilometres, and have raised the accordant concentration levels by about three orders of magnitude. The two stations CNX22 in Hong Kong and JPX38 near Tokyo have taken data, with which it has been possible to gather further information about the Fukushima emissions. The time series of detections has a similar slope to the decay of ${ }^{133} \mathrm{Xe}$, so that a memory effect in the detector cannot be excluded. This would lead to an overestimation of the source term.

The presented method allowed gathering detailed information about the Fukushima ${ }^{133}$ Xe source term in a time resolution equal to the detector's sampling time. When reconstructing the Fukushima ${ }^{133}$ Xe source term and including previously established ${ }^{137} \mathrm{Cs}$ and ${ }^{131}$ I source terms, it is seen that they correlate with external observations of the events such as the appearance of radionuclide peaks with reported explosions and smoke. In terms of magnitude the estimated source terms for ${ }^{133} \mathrm{Xe}$ are in good agreement with other published data (Stohl et al., 2011). In terms of time dependence a reasonable agreement with previously established source terms of ${ }^{137} \mathrm{Cs}$ and ${ }^{131} \mathrm{I}$ is found. Furthermore, the results suggest that this is a valid method for remotely determining source terms.

\section{Acknowledgments}

The authors highly acknowledge the support by the National Scientific Committee Technology of INFN for the ERMES project, and the European Commission under the FP7 programme for the EUMEDGRID project (Grant RI-246589). The authors are also grateful to the Grid Lab of INFN and the Department of
Mathematics and Physics of University of Roma Tre, and specifically to Federico Bitelli and Antonio Budano. The atmospheric transport modelling has been accomplished with meteorological data provided by the European Centre for Medium-Range Weather Forecasts under a collaboration agreement with the University of Roma Tre.

\section{References}

Auer, M., Kumberg, T., Sartorius, H., Wernsperger, B., Schlosser, C., 2010. Ten years of development of equipment for measurement of atmospheric radioactive xenon for the verification of the CTBT. Pure Appl. Geophys. 167, 471-486.

Hirose, K., Aoyama, M., Povinec, P.P., 2012. Radiostrontium in the western North Pacific: characteristics, behavior and the Fukushima impact. Environ. Sci. Technol., submitted.

IAEA (International Atomic Energy Agency), 2011. Fukushima Nuclear Accident Update. 〈http://iaea.org/newscenter/news/tsunamiupdate01.html〉.

Kalinowski, M.B., Tuma, M.P., 2009. Global radioxenon emission inventory based on nuclear power reactor reports. J. Environ. Radioact. 100, 58-70.

Plastino, W., Plenteda, R., Azzari, G., Becker, A., Saey, P.R.J., Wotawa, G., 2010. Radioxenon time series and meteorological pattern analysis for CTBT event categorisation. Pure Appl. Geophys. 167, 559-573.

Schoeppner, M., Plastino, W., Povinec, P., Wotawa, G., Bella, F., Budano, A. De Vincenzi, M., Ruggieri, F., 2011. Estimation of the time-dependent radioactive source-term from the Fukushima nuclear power plant accident using atmospheric transport modeling. J. Environ. Radioact. , http://dx.doi.org/ 10.1016/j.jenvrad.2011.11.008.

Stohl, A., Hittenberger, M., Wotawa, G., 1998. Validation of the Lagrangian particle dispersion model Flexpart against large-scale tracer experiment data. Atmos. Environ. vol. 32 (24), 4245-4264 1998

Stohl, A., Sodemann, H., Eckhardt, S., Frank, A., Seibert, P., Wotawa, G., 2010. The Lagrangian Particle Dispersion Model FLEXPART, Version 8.2. Norwegian Institute of Air Research, Kjeller, Norway, 〈http://transport.nilu.no/flexpart〉.

Stohl, A., Seibert, P., Wotawa, G., Arnold, D., Burkhart, J.F., Eckhardt, S., Tapia, C., Vargas, A., Yasunari, T.J., 2011. Xenon-133 and caesium-137 releases into the atmosphere from the Fukushima Dai-ichi nuclear power plant: determination of the source term, atmospheric dispersion, and deposition. Atmos. Chem. Phys. Discuss. 11, 28319-28394.

Wotawa, G., De Geer, L.E., Denier, P., Kalinowski, M., Toivonen, H., D'Amours, R. Desiato, F., Issartel, J.P., Langer, M., Seibert, P., Frank, A., Sloan, C., Yamazawa, Y., 2003. Atmospheric transport modelling in support of CTBT verification-overview and basic concepts. Atmos. Environ. 37, 2529-2537.

Wotawa, G., Becker, A., Kalinowski, M., Saey, P., Tuma, M., Zaehringer, M., 2010. Computation and analysis of the global distribution of the radioxenon isotope 133Xe based on emissions from nuclear power plants and radioisotope production facilities and its relevance for the verification of the Nuclear-TestBan Treaty. Pure Appl. Geophys. 167, 541-557.

Zähringer, M., Becker, A., Nikkinen, M., Saey, P., Wotawa, G., 2009. CTBT radioxenon monitoring for verification: today's challenges. J. Radioanal. Nucl. Chem. 282, 737-742. 